This item was submitted to Loughborough's Research Repository by the author.

Items in Figshare are protected by copyright, with all rights reserved, unless otherwise indicated.

\title{
Educating the world: a remote experiment in photovoltaics
}

PLEASE CITE THE PUBLISHED VERSION

http://dx.doi.org/10.1109/ICONCE.2014.6808676

PUBLISHER

(c) IEEE

VERSION

AM (Accepted Manuscript)

LICENCE

CC BY-NC-ND 4.0

REPOSITORY RECORD

Blanchard, Richard E., Sheryl R. Williams, Martin Bliss, Michael S. Clowes, A. Mohammed, and Ramesh Pancholi. 2019. "Educating the World: A Remote Experiment in Photovoltaics". figshare.

https://hdl.handle.net/2134/15422. 


\title{
Educating the World: A Remote Experiment in Photovoltaics
}

\author{
R. Blanchard ${ }^{1}$, S. Williams. M. Bliss, M. Clowes, A. Mohammed and R. Pancholi \\ School of Electronic, Electrical and Systems Engineering \\ Loughborough University \\ Loughborough, Leicestershire, United Kingdom, LE11 4NU \\ ${ }^{1}$ r.e.blanchard@lboro.ac.uk
}

\begin{abstract}
The increasing deployment of photovoltaic (PV) systems requires large numbers of skilled engineers with a greater understanding of all aspects of PV technology both theoretical and practical. Developing experimental rigs at universities is expensive and limited to students physically attending the university. One recent approach to increase access to laboratories is the development of remote experiments. Here students can control real experimental equipment using a visual interface via the Internet. In this paper we explore the development of a photovoltaic laboratory to enable users to access and remotely control experimental equipment based at Loughborough University, UK, from anywhere in the world.
\end{abstract}

Index Terms - photovoltaic, remote laboratory, IV curves, MPP, distance learning.

\section{INTRODUCTION}

Photovoltaic (PV) devices convert light energy to electrical energy for use in various applications. Therefore, it is important that the energy conversion characteristics of a specific PV device is fully understood for a range of operational conditions. One method for assessing PV performance is by measuring the "characteristic" (or IV) curve. An experiment that produces IV curves of different PV technologies under varying light conditions including the effect of temperature on cell performance has been used by MSc Renewable Energy Systems Technology students at Loughborough University since the course began in 1994. The experiment has been updated as required. However, students only have a limited time to use the equipment and there a limited number of test rigs available. Figure 1 shows a schematic of the experimental set-up of the optical bench

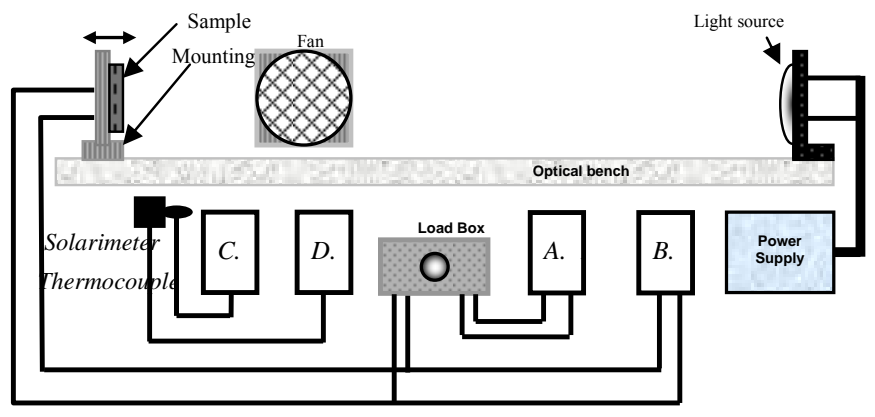

Fig. 1. Physical laboratory set-up
There has been debate about the role of laboratories in engineering education [1]. The normal form of conducting experiments involving physical presence is generally termed as hands on laboratory. Recent developments in information and communication technologies during the last few decades resulted in new technologies like Internet based labs and simulated labs. These online labs allow remote access to the physical experimental system through a Intranet/Internet connection. The first idea of implementing laboratories through the Internet for educational purposes was started in early 1990's [2]. Since then the number of Internet based laboratories have been increasing, for example an outdoor solar laboratory for schools [3]. The possibility of sharing resources with other institutes, hence gaining economical benefits is an advantage of the remote laboratories. Another important thing to note is that, using real hardware is the only thing to engage students and help them to comprehend their engineering studies. Some advantages of using remote laboratories are: (a) Reduce the number of student hours spending in the laboratory by increasing our self-paced learning offering, (b) Achieving practical experiences with real equipment through Internet connection from home, (c) Giving access to more learning materials, procedures and practical video presentations through e-learning, (d) Monitoring on real time measuring system by using a web camera and storing the acquired data. Students welcome remote laboratories because they increase access flexibility, so they are a growing in number across the world [1], [4-8]. They are particularly beneficial to distance learning students as it avoids time and costs required to attend a physical laboratory at a given location.

The development of a remote controlled laboratory presented a number of challenges. To replicate the physical apparatus shown in figure 1, a requirements capture exercise identified a number of sub-system requirements that would be critical to the functionality of the experiment.

- Create a Light source which can be remotely controlled to change the level of irradiance.

- Create a sub-system which will allow the changing of PV panels remotely.

- Create a sub-system to measure irradiance.

- Create a sub-system which will measure and control the temperature of the PV panels remotely. 
- Connect and integrate the complete system to a computer which will allow the system to be accessed and controlled over the internet by users.

The aims of the paper are to present the results of the development of a remote PV experiment to show the potential for training technicians and engineers around the world. The paper outlines the requirements capture, the design of the experimental set-up, the need to develop a software interface between the remote user and hardware and presents the findings and future development of the project.

\section{METHODS}

One of the challenges of developing a remote PV laboratory is that users are unable to physically alter experimental parameters. For example, in the physical lab to alter the irradiance hitting the PV sample panel is carried out by moving the panel towards or away from the light source. In addition to an experimental rig that would function as the apparatus in Figure 1, a user control interface would be required as shown in Figure 2.

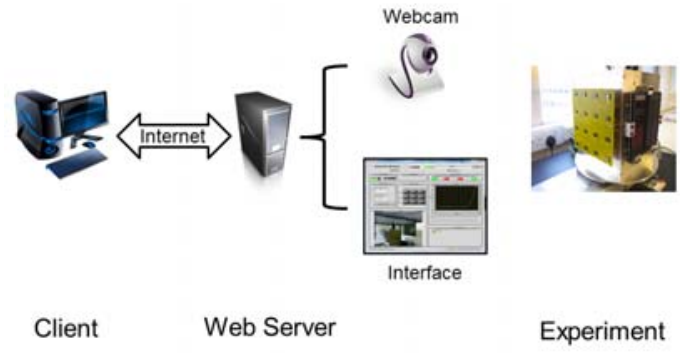

Fig. 2. Remote laboratory example diagram

Therefore, the design of the experiment required automation controls that could be operated at a distance without need for human intervention. In addition, specific design parameters were required to meet the experimental test requirements namely:

- Measure and plot the characteristic curves for a range of PV cell types under various irradiance and temperature conditions;

- Quantify the maximum power point (MPPT), fill factor (FF) and conversion efficiency for each technology;

- Compare and contrast the performance characteristics of the different types of photovoltaic samples;

- Assess how the nature of the light source affects the results for different PV technologies;

- Perform STC corrections on the data;

- Specify reasons for any discrepancies between the experimental and 'ideal' STC results;

- Describe the relevance of the results to the performance of PV systems in real applications.

The irradiance and temperature parameters were specifically:

At a temperature of $\sim \mathbf{3 0}^{\circ} \mathbf{C}$ measure current \& voltage from open circuit to short circuit for varying load resistances at the following values of irradiance. $\sim 250 \mathrm{~W} / \mathrm{m}^{2}, \sim 500 \mathrm{~W} / \mathrm{m}^{2}$ and $\sim 750 \mathrm{~W} / \mathrm{m}^{2}$
At an irradiance of $\sim 500 \mathrm{~W} / \mathrm{m}^{2}$, measure current \& voltage from open circuit to short circuit for varying load resistances at the following temperatures:

$$
\sim 30^{\circ} \mathrm{C}, \sim 40^{\circ} \mathrm{C} \text { and } \sim 50^{\circ} \mathrm{C}
$$

\section{A. Description of Experimental Set-up}

The hardware requirements to meet the experimental parameters led to two main design solutions, a controllable variable light source and a controllable temperature device to maintain PV panel temperature at the desired level. These solutions are described in the following sections.

\section{1) Light Source:}

Light Emitting Diode (LED) modules were used as they were specifically designed to replicate the spectrum of sun light which was fundamentally important in this application of testing PV devices. In addition, the LED produces differing levels of irradiance when a variable power source is available. A total of 36 LEDs modules were arranged in a six-by-six array as shown in figure 3 . These were connected to a PV simulator LED current regulation board. The voltage source for these LEDs was provided by a TDK Lamda programmable voltage source module which provided voltage flow through the LED modules. The LED array was fixed to a heat sink to allow continuous operation without the LED module overheating and allow efficient heat dissipation. Fans were then attached to the back of these fins for further cooling.

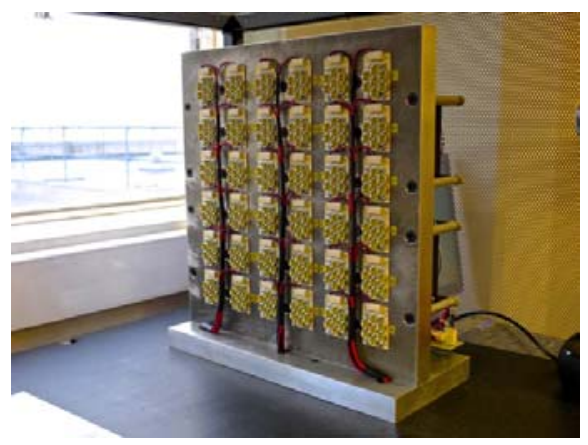

Fig. 3. LED Array.

Irradiance levels for the LED array were measured using an array of $164 \times 4$ photodiodes. To record a measurement of the levels of irradiance, a photodiode rig was built and set up alongside the PV panels on a rotating platform. The hotodiodes used are visible to IR photodiodes. This set up allows users to measure the actual irradiance the LEDs are producing to use in the calculations. The photodiodes were built onto a circuit board which is shown in figure 4 . The circuit allows the photodiode to send voltage readings to the computer which converts it into a level of irradiance in Watts per square metre. This will be the value that the users will use when making further calculations with the data when testing the PV panels.

\section{2) Light Source:}

To ensure that the irradiance measured by the photodiode array was the same as that received by the test PV sample 
panels, the PV panels and photodiode array were attached to a rotating platform on base platform for stability as shown in figure 4. A stepper motor was used to rotate the panel / photodiode arrangement.

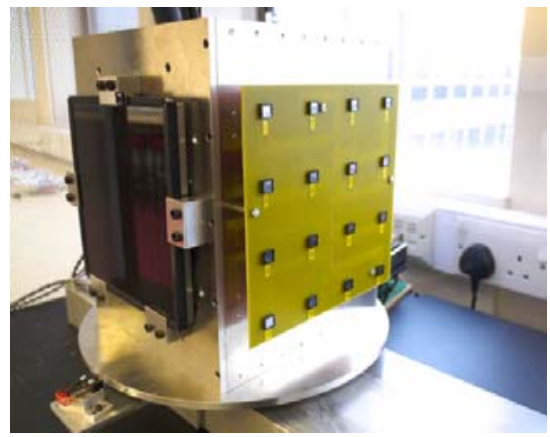

Fig. 4. PV panel / photodiode rotating platform.

Another variable in the experiment for the users to control is the temperature of the panels. This means that the temperature of the panel needs to be controlled and shown to the user. The control of this experimental variable was performed by a Peltier module (heating/cooling plate) arrangement on the back of the PV panels. In the experiment there were three different temperatures at which readings needed to be taken namely; $30^{\circ} \mathrm{C}, 40^{\circ} \mathrm{C}$ and $50^{\circ} \mathrm{C}$. The Peltier was linked into the LabVIEW programme which will be the control of the temperature. The measurement of the temperature of the Panels was taken by a PT100 temperature sensor which is placed on the back of the panel so that the heat from the LEDs does not affect the temperature reading. The panel was attached with a thin layer of conducting gap filler which also allows the temperature sensor and output cable of the panel to be placed between the panel and the aluminium plate. This again was linked to the computer using the DAQ card. The arrangement for the PV panel temperature control is shown in figure 5 .

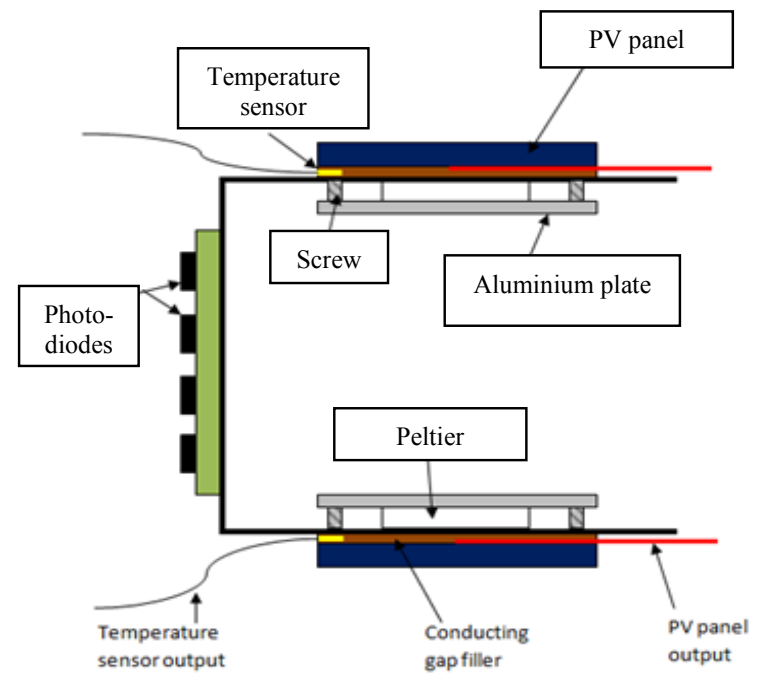

Fig. 5. Top view of temperature control and measurement configuration design.

\section{B. Programming Design}

As the hardware configuration was being constructed, the software to enable users to perform the experiments was developed. Bearing in mind the apparatus consists of the LED based lighting system for artificial irradiance, heating/cooling peltiers for the PV modules, stepper motor to change panel using turn table, temperature sensors for measuring temperature on the panel, programmable power supplies, a Keithley device for measuring current $\&$ voltage, a base for placing LED board \& PV module, irradiance measurement sensors, GPIB cable and DAQ card with connection ports. There would be need to integrate this equipment to find a software solution to enable the experiment to work and allow for remote use.

There are different types of tools available in the market to implement these online laboratories [9]. However, the LabVIEW software environment has been demonstrated to be suitable for remote control of equipment [1] [10]. Indeed it includes tools for designing automation control in engineering [11]. An additional factor in the selection of LabVIEW is the ability to publish to the web, which is crucial to the use of remote laboratories, through the Internet Toolkit facility.

From the user perspective the virtual instrument (VI) front panel gives the students the ability to control the experiment. Once the main LabVIEW programs were written, interfaced with the hardware, validated and integrated, next step was the remote connection facility. The development of this functionality is described in the following section.

\section{1) Virtual Instrument:}

The front panel is the interactive graphical user interface (GUI) of a VI, named because it looks similar to the front panel of a physical instrument. Controls resemble the physical buttons, knobs and switches that can be found on a conventional instrument. The controls need data or a response from a user to supply the block diagram for processing. The indicators display the generated data for users received from the LabVIEW program outputs. Behind the VI is the programme block diagram that contains the main VI's source code developed using the LabVIEW programming language $\mathrm{G}$, which is similar to $\mathrm{C}++$ or Java.

\section{2) Hardware interfacing}

10 different programmes would be required to run the experiment that would also integrated into the VI GUI. The programmes to be developed were:

- Power control for irradiance

- Power control Peltier device

- Programme control for irradiance

- Programme control for Peltier device

- Irradiance measurement

- Temperature measurement

- IV curve measurement

- Photodiode / PV Panel motor control programme

- Data Storage

- Web Camera 


\section{3) Hardware interfacing}

Figure 2 indicates the additional Client and Web server requirements of the remote laboratory. The procedure for a remote connection to the VI is; first an HTML code implemented by the LabVIEW Web publishing tool embeds the VI on the server. This HTML code is available in LabVIEW web server root directory. After closing this window, the access to the VI remotely is established and the web server is activated. The front panel can be accessed via the server name. The LabVIEW web server and the LabVIEW runtime engine are compiled in the installation kit.

When a client requests access to the VI through a webpage which includes the HTML code of the embedded VI of the laboratory, the VI would upload to the client's PC. Hence, the user is remotely connected to laboratory through the LabVIEW web server. The requirement for this web access is to install a LabVIEW runtime engine on the user PC. This software is available to download at free of cost from the NI website. This process is much similar to many Internet embedded applications which require installation of their own soft-wares to enable the functionality of the application like Flash files, or Java applets PC. The experiment can be observed through a web camera connected to the server. The camera makes use of a programme that is part of LabVIEW.

\section{RESUlTS AND DISCUSSION}

A considerable amount of work effort went into the construction of the laboratory from the hardware components and software programming. The deliverables consisted of individual elements interfaced into the project package.

Controller programmes were successfully developed, for example figure 6 illustrates one of these, namely the photodiode measurement programme. Interestingly the user does not see these programmes as they are behind the front panel VI GUI shown in figure 7.

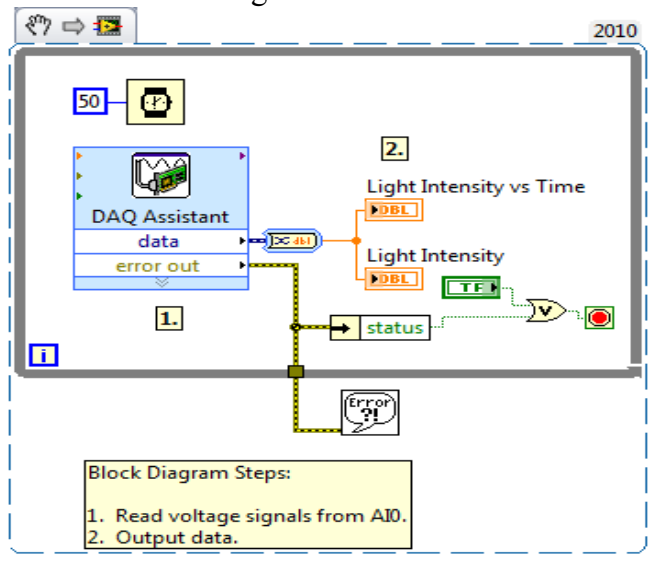

Fig. 6. Measuring Irradiance from photo diode

The GUI VI front panel, shown in figure 7 demonstrates what the remote user will see when they access the remote laboratory. There are two arrays of voltage and current values from the Keithley source meter. And using the same arrays a waveform graph is plotted. There are indicators for irradiance and temperature on front panel showing exact values. Two further indicators are used to show whether the required irradiance and temperature are reached or not. A window for the web-camera is placed at the corner of the front panel. A timer is place to show elapsed time. The VI experiment is limited to 2 hours and therefore stops after this time. A booking system was developed to allow for users to pre-book their times on the laboratory [12]. A switch is placed to acquire data when the user wants to measure an I-V curve. Finally another switch allows the user to change the rotating platform between the photodiode and PV panels to allow the user to complete the experiment.

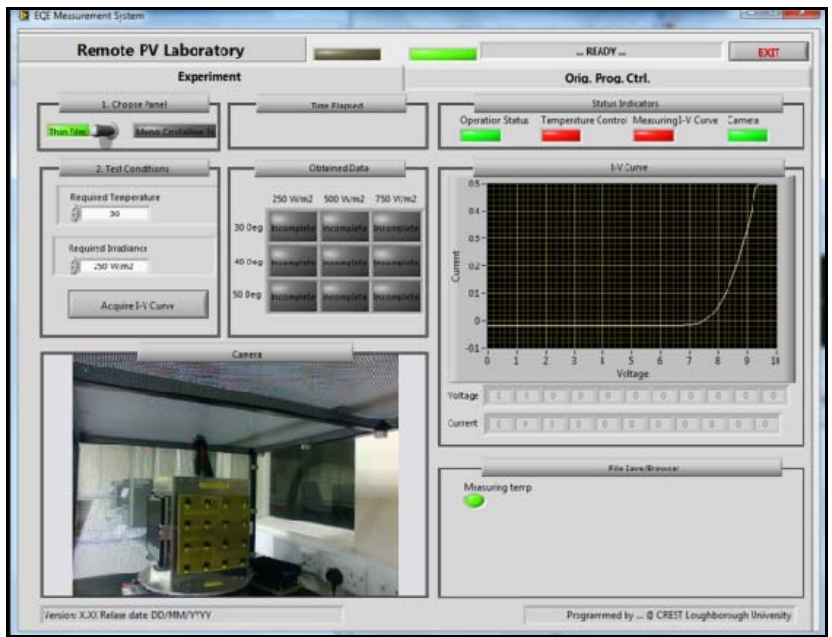

Fig. 7. Graphical User Interface for Remote Laboratory.

The development of the laboratory was not without its challenges. Whilst the project funding paid for the majority of the experimental hardware, it did not include the bench nor the bespoke LED heat sink and PV devices turntable. Support for this was provided by the School with materials, and mechanical and electronics technician's time. A diverse range of skills was needed. To enable Internet access to the experiment a secure network port had to be opened. Figure 8 shows the apparatus in use.

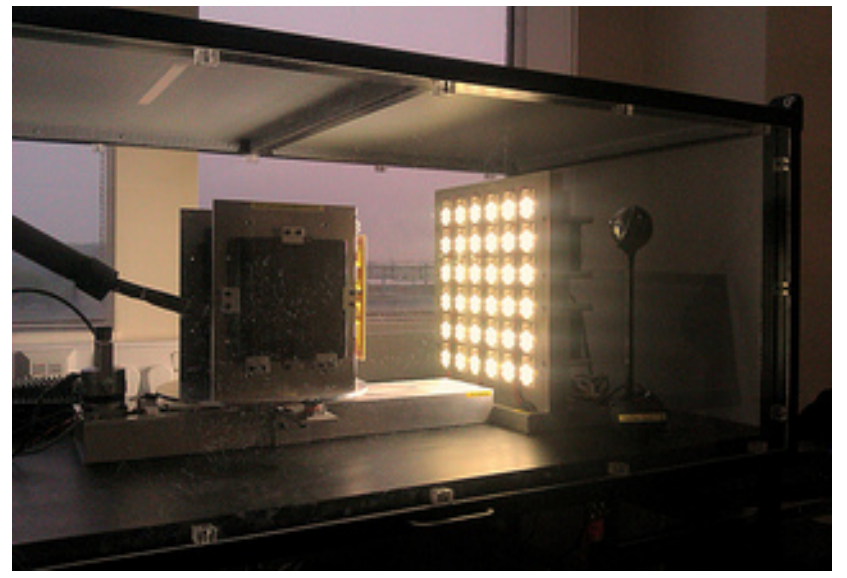

Fig. 8. Remote photovoltaic experiment apparatus. 


\section{CONCLUSIONS}

A remote access experiment was designed and developed to enable users to access apparatus to permit learning about the characteristics of different PV materials. The project developed a rig with hardware components that could be programmed using LabVIEW to respond to user commands via the Internet. The student's results from the experiment can be exported and downloaded for interpretation in a summative assessment. A booking system allows users to access the remote laboratory at a chosen time and avoids the possibility of more than one user accessing the rig.

There were challenges that were dealt with. This is a complicated experiment incorporating, hardware, software, electronics, control, automation and measurement. Not to mention remote Internet access.

The remote laboratory developed in Electronic Electrical and Systems Engineering, Loughborough University is not only an attractive tool of teaching measurement technology for students but it also has industrial importance with the deployment of photovoltaic technologies around the world. At present globalised students often travel from one side of the globe to the other to study and to improve their knowledge on the use or operation of a system. In contrast, distance learning students could study from their homes and control experiments in laboratories via the Internet without this need to travel. This remote experiment technology would improve their practical knowledge and also allow for students to repeat the experiment at a time to suit their needs. This is often not feasible in a face-to-face physical laboratory [1]. Future work will involve evaluating the student experience.

\section{ACKNOWLEDGMENTS}

The project was part-financed by a Loughborough University Distance Learning Enhancement Fund.

\section{REFERENCES}

[1] M. Abdulwahed., Towards Enhancing Laboratory Education, $\mathrm{Ph} . \mathrm{D}$.Thesis, Loughborough University, United Kingdom, Jun 2010.
[2] ASEE (2010) The American society for engineering education. [Online] Available from: www.asee.org [Accessed 20 January 2010].

[3] W. Hutzel., Remotely accessible solar energy laboratory for high school students. In Proc. Frontiers in Education Conference, 3, 18-22, 2004.

[4] Pavol Bauer, Viliam Fedák and Otto Rompelman: PEMCWebLab - Distance and Virtual Laboratories in Electrical Engineering: Development and Trends, 13th International Power Electronics and Motion Control Conference (EPE-PEMC 2008), 1-3 September 2008, Poznan, IEEE Catalog Number: CFP0834A-CDR, ISBN: 978-1-4244-1742-1.

[5] Angéla Váradiné Szarka, Development of remote controlled virtual laboratory, XIX IMEKO World Congress Fundamental and Applied Metrology, September 6-11, 2009, Lisbon, Portugal.

[6] P. Bauer, V. Fedak: Distance Laboratories in Electrical Engineering, International conference EDPE Stara Lesna Slovakia, September, 2007, ISBN 978-8073-867-9.

[7] Aktan, B., Bohus, C. A. \& Shor, M. H. (1996) Distance learning applied to control engineering Laboratories. IEEE Transactions on Education, 39(3), 320-326.

[8] P. Coquard, et al., 2008, "Remote Laboratories: AIP-Primeca RAO Platform".

[9] T. Machino, S. Iwaki, H. Kawata, Y. Yanagihara, Y. Nanjo \& K. Shimokura, "Remote Collaboration System using Mobile Robot with Camera and Projector", vol. Proceedings of the 2006 IEEE International Conference on Robotics and Automation, no. May 2006.

[10] Li Pengfei, Nie Luhua, Remote control laboratory based on Labview, 2009 2nd International Conference on Intelligent Computation Technology and Automation, 2009.

[11] P. Bauer, V. Fedak: Distance Laboratories in Electrical Engineering, International conference EDPE Stara Lesna Slovakia, September, 2007, ISBN 978-8073-867-9.

[12] S. Uran, D. Hercog, K. Jezernik: Remote Control Laboratory with Model Booking System. In Proceedings of the IEEE International Symposium on Industrial Electronics (ISIE), 2007, pp. 2978-2983. 